\title{
Engrasando la maquinaria legislativa: precedentes y dosieres legislativos
}

\author{
Adjusting the legislative machine: legislative precedents and dossiers
}

\author{
Virginia Ramírez Martín (1), Gloria de La Chica Blanco (2)
}

(1) Departamento de Archivo del Congreso de los Diputados, Floridablanca s/n 28071 Madrid, virginia.ramirez@congreso.es. (2) Departamento de Documentación del Congreso de los Diputados, gloria.chica@congreso.es

\begin{abstract}
Resumen
Los precedentes y dosieres elaborados por los servicios documentales de los parlamentos son un medio auxiliar de enorme utilidad en el procedimiento de elaboración de las leyes. A partir de una perspectiva eminentemente práctica, la presente comunicación tiene por objeto presentar estos productos documentales centrando sus objetivos, metodología y principales destinatarios.
\end{abstract}

Palabras clave: Servicios documentales parlamentarios. Procedimiento legislativo. Archivística. Biblioteconomía. Documentación legislativa. Cortes Generales. Instituciones legislativas nacionales. España.

\section{Introducción}

Que el proceso de elaboración de las leyes necesita de información adecuada, pertinente y objetiva es algo de lo que eran ya conscientes los diputados doceañistas. Por ese motivo, las Cortes Extraordinarias reunidas en Cádiz decretan el 24 de enero de 1811 "que se reúnan todas las Actas y Cuadernos de Cortes, desde las más antiguas, todos los Fueros, Ordenanzas, Cuerpos Legales, y en suma todas las obras tocantes a la legislación española". La finalidad de reunir todas estas obras, y muchas más que se fueron añadiendo, era crear una colección de documentos que pudiera servir a la instrucción pública pero, más señaladamente, para el uso del Congreso Nacional, pues ya entonces afeaba Bartolomé José Gallardo, lexicógrafo y a la sazón Bibliotecario de Cortes primero y más tarde del Congreso de los Diputados (Gallego, 2006), la conducta del que "creyese buenamente que para hacer leyes no son menester libros ni bibliotecas". A ello se une también muy pronto la existencia de un Archivo de Cortes, de modo que se conservase memoria fiel de la actividad del cuerpo legislador.

Desde entonces hasta ahora han cambiado los medios, la naturaleza de los documentos y su accesibilidad, pero sigue presente el fin y el espíritu que mueve la labor de los archiveros-bibliotecarios parlamentarios, que es colaborar con su

\begin{abstract}
Precedents and dossiers developed by the document services of Parliaments are both a very useful tool in the Legislative Process. From a practice point of view, this paper tries to show these information products from the study of their goals, methodology and users.
\end{abstract}

Keywords: Legal documentation. Archival science. Library science. Information services. Legislative processes. National legislative bodies. Congress. Spain.

trabajo para que el legislador tenga a su disposición toda la información necesaria para elaborar mejores leyes. Los documentos como materia y la información como esencia han estado, pues, muy unidos a la actividad parlamentaria y, muy especialmente, a la que es la función primordial de los parlamentos: la función legislativa. La elaboración de las leyes, cuyo cauce es el procedimiento legislativo, es un proceso reglado, pues en todo se atiene a lo que marca el texto constitucional y los reglamentos de las cámaras legislativas. En el caso español, tanto uno como el otro han sido modificados con profusión a lo largo del tiempo, pues son muchas las Constituciones que han jalonado nuestro siglo XIX y en menor medida $X X$, y también las normas de funcionamiento del poder legislativo, tanto en los períodos en los que se ha establecido que fuera uni o bicameral, han sido revisadas con cierta asiduidad.

La provisión de la documentación e información necesarias puede insertarse como útil o medio auxiliar en lo que se conoce como técnica legislativa que, en palabras de Piedad García-Escudero constituye el arte de legislar clara y eficazmente y, como tal, forma parte de la ciencia de la legislación (García Escudero, 2005). Como bien señala la autora, citando en este caso a Fernando Sainz-Moreno (ibídem, p. 122),

El objeto de la técnica legislativa no es sólo la buena redacción de las leyes, sino que afecta a cuestiones 
más generales y trascendentes, como son la unidad y coherencia del ordenamiento jurídico y la calidad, la publicidad y la viabilidad de las normas.

En la actualidad los servicios documentales de los parlamentos, y este es también el caso del Congreso de los Diputados, atienden las necesidades de información de diversos grupos de usuarios, aunque, sin duda, el principal es el de los diputados, los asistentes de los grupos parlamentarios y los funcionarios de la propia cámara. A ellos se destina en primera instancia la elaboración de diversos productos documentales que tienen por objeto auxiliar en las labores que son propias de su actividad, a saber: la función legislativa y la función de control del ejecutivo.

En la presente comunicación se propone un acercamiento a los productos documentales elaborados por los Departamentos de Archivo y Documentación del Congreso de los Diputados que tienen como principal finalidad apoyar la función legislativa, y que son fundamentalmente los precedentes y los dosieres legislativos. Esta aproximación se pretende plantear desde una perspectiva profesional centrada en el análisis de dos productos documentales específicos, pues la documentación parlamentaria ya ha sido abordada por diversos estudios tanto de alcance genérico como especializado.

Asimismo, conviene subrayar que estos productos nacen de la vocación de difusión propia de los servicios documentales y de la función de asistencia y estudio que, como se verá a continuación, tienen encomendada los profesionales de la documentación, los archivos y las bibliotecas que prestan servicio a los parlamentos, y para ello se trata de presentar una versión conjugada de los procedimientos documentales en los que intervienen los departamentos de archivo y documentación.

El objetivo de estas líneas no es otro que dar a conocer, fuera del ámbito parlamentario, los citados productos documentales (precedentes y dosieres legislativos), la forma en la que se elaboran, cuáles son sus destinatarios y los casos en los que son solicitados o se elaboran de oficio y subrayar la importancia que tienen en el ámbito de la técnica legislativa en tanto auxiliares de aquella.

\section{Los servicios documentales del Congreso de los Diputados}

Los servicios documentales de los parlamentos, con independencia de su organización material, aglutinan el archivo de la cámara legislativa, la biblioteca y el centro de documentación que pueden ser unidades independientes o no en función de las dimensiones de la cámara, su tradición y otro tipo de cuestiones de índole organizativa.
En el caso del Congreso de los Diputados y atendiendo al artículo 60.1 de su reglamento, se dice que este "dispondrá de los medios personales y materiales necesarios para el desarrollo de sus funciones, especialmente de servicios técnicos, de documentación y de asesoramiento". Asimismo, el artículo 8.2 del Estatuto del personal de las Cortes Generales dispone que corresponde al Cuerpo de Archiveros-Bibliotecarios

[...] desempeñar las funciones de organización y gestión de los recursos documentales y bibliográficos existentes en la cámara, así como el cuidado y conservación del patrimonio documental y bibliográfico de las Cortes Generales; la realización de las tareas de asistencia y asesoramiento, informe, estudio y propuesta de nivel superior en las materias que le son propias; la jefatura de los servicios correspondientes y el acceso a la función de dirección en las materias propias de su especialidad según se determine en las plantillas orgánicas.

Esto establece, en resumen, una reserva material de funciones para el Cuerpo de ArchiverosBibliotecarios, garantizando que este servicio se preste por el personal que tiene la cualificación necesaria para ello, al tiempo que se consagra la importancia de estas funciones al disponer de un cuerpo especializado que está en condiciones de prestarlas.

Es necesario, al mismo tiempo, hacer mención a otro de los extremos que se explicitan en el artículo $58 \mathrm{~g}$ ) del citado Estatuto, por el que se establece, entre las obligaciones de los funcionarios de las Cortes Generales, la de "actuar con absoluta imparcialidad política en el cumplimiento de su función y abstenerse de actuación política dentro de las cámaras".

Esta previsión y la ética profesional que rige la actuación en el marco de estas funciones, así como las peculiaridades que imprime a nuestro trabajo dos siglos de tradición documental en el ámbito de las Cortes son la guía de una actividad que tiene como principal mandato el de la realización de las funciones que los funcionarios del Cuerpo de Archiveros-Bibliotecarios tienen encomendadas en virtud del Estatuto y que se traducen, en muchos casos, en una marcada actividad de difusión de la información y del patrimonio documental y bibliográfico del que somos custodios.

En lo que atañe a la organización administrativa, se dirige desde la Dirección de Documentación, Biblioteca y Archivo dependiente, a su vez, de la Secretaría General Adjunta para Asuntos Parlamentarios. La citada dirección comprende los tres departamentos que le dan nombre: Documentación, Biblioteca y Archivo. Los servicios documentales del Congreso de los Diputados se integraron, inicialmente, en la Dirección de Estudios y Documentación, si bien en 2007 se desgajó 
esta, existiendo desde entonces dos Direcciones: la de Estudios y Publicaciones y la de Documentación, Biblioteca y Archivo.

\section{El Departamento de Archivo}

El Archivo del Congreso de los Diputados, al que generalmente se alude en las notas al pie de página de muchas publicaciones al citar las fuentes primarias como ACD, es uno de los departamentos que integran la Dirección de Documentación, Biblioteca y Archivo.

Su fondo lo conforma la totalidad de los documentos generados por la cámara en su actividad, tanto parlamentaria como administrativa, a lo largo de su historia. Desde 1808 hasta hoy, todos los órganos y dependencias han transferido, y lo siguen haciendo, su documentación al archivo de la cámara. El antiguo Archivo de Cortes es, por lo tanto, el germen del actual Archivo del Congreso de los Diputados que conserva también la documentación generada en los períodos en los que el poder legislativo ha sido unicameral (1810$1814,1820-1823$ y 1931-1939). Así, esta documentación es fiel reflejo de la historia y actividad del poder legislativo en España y, junto con el Archivo del Senado, es de consulta inexcusable para conocer, entre otras cosas, el origen y la evolución de parlamentarismo en nuestro país.

El archivo ha vivido las trashumancias de las Cortes hasta su establecimiento definitivo en Madrid $y$, después, su instalación en el nuevo palacio hasta el traslado a su ubicación actual en la Ampliación I del complejo del Congreso de los Diputados. La organización actual de sus fondos es deudora de los criterios establecidos en 1841 por Clemente Arias aunque desde 1982, momento en el que se separan definitivamente el archivo y la biblioteca, se determina el esquema actual de secciones y series.

Su norma de funcionamiento es poco posterior a esta fecha, pues la Instrucción sobre el Archivo del Congreso de los Diputados data del 4 de julio de 1984. En ella se detalla su definición, alcance, obligatoriedad de transferencia de la documentación por parte de todos los órganos y servicios productores, así como el régimen de acceso a la misma. Esta norma se ha visto parcialmente modificada en diversos aspectos; el más reciente tiene que ver con la entrada en vigor de la Ley de Transparencia y la modificación de la norma sobre acceso y valoración de fondos documentales y bibliográficos.

Actualmente el Departamento de Archivo se organiza en dos Servicios: el de Difusión de Información y Difusión de Fondos Archivísticos y el de Ingreso y Tratamiento de Fondos Archivísticos, que incluye a su vez la Unidad de Fondos Parlamentarios y la de Fondos Administrativos.

\section{Precedentes: acotando el concepto}

Precedente se dice de aquello que antecede a otra cosa en el tiempo, y que asimismo, por ser semejante o de su misma clase, influye o sirve de ejemplo.

Esta definición permite acotar un concepto complejo y polisémico que, en lo que atañe a esta comunicación, se circunscribe esencialmente a los precedentes legislativos y los precedentes reglamentarios, dejando fuera de la cuestión otro tipo de precedentes a los que se acude también de manera recurrente y con multitud de propósitos.

El precedente legislativo tiene por objeto ilustrar la elaboración de una norma con rango de ley que se va a acometer, haciendo un repaso no solo por la norma vigente, la inmediatamente anterior, si es que existe, hasta el momento en el que se va a proceder a su reforma y también por la recopilación de otras iniciativas legislativas y debates parlamentarios que guardan relación con la cuestión. Se trata, pues, de rastrear en el ámbito parlamentario las raíces de una cuestión para abordar su tratamiento legislativo.

Por otra parte, el precedente que aquí se ha llamado reglamentario o parlamentario afecta al modo en que se ha aplicado el reglamento de la cámara en atención a circunstancias similares. Este es un precedente que está más próximo al precedente administrativo $\mathrm{y}$, como sucede también con él, no es algo que ligue la actuación futura, si bien indica cómo se ha obrado en una circunstancia determinada, aunque separarse de aquel implica la necesidad de justificar debidamente la actuación.

En cualquiera de los dos casos es el Archivo del Congreso de los Diputados, memoria y registro de la actividad de la cámara a lo largo del tiempo, quien custodia la documentación en la que obra la información que atañe a los precedentes, sean del tipo que sean. $Y$ es también el archivo el departamento encargado de la elaboración de los productos documentales independientes en los que se materializan los precedentes.

\subsection{El precedente legislativo}

Como antes se indicaba, precedente es un concepto polisémico que, en primera instancia, se habrá de analizar circunscrito a su intersección con el adjetivo legislativo. En este sentido pueden ser muchos los ejemplos que ayuden a ilustrarlo, pero se citarán dos: uno de ellos en los que no existe norma previa y otro en el que sí. 
El primer caso, aquel en el que no existe ordenamiento jurídico a reformar sino que se plantea una norma ex novo puede ser el de la tramitación parlamentaria de la que después será la Ley 12/2015, de 24 de junio, en materia de concesión de la nacionalidad española a los sefardíes originarios de España.

Bien es cierto que esta norma no tiene precedente en nuestro ordenamiento, y tampoco en el ámbito de la legislación comparada, pero sí que eran pertinentes los debates surgidos en torno a la denominada entonces "cuestión judía". Un somero repaso por la cuestión reveló que el derecho al retorno de los sefardíes que fueron expulsados de España en 1492 había sido abordado en sede parlamentaria a principios del siglo $X X$, como consecuencia de las campañas emprendidas por el doctor Pulido en el norte de África, donde se encontraban colonias de sefardíes que se habían instalado allí tras la expulsión de los judíos de la Península en el siglo XV. Aquellos pobladores conservaban su lengua, el ladino o judeoespañol, y mantenían viva la cultura que habían desarrollado en España. Sin embargo, este redescubrimiento de lo sefardí es previo, pues a mediados del siglo XIX se comienza a abordar, especialmente en lo tocante a la libertad religiosa y su consideración en el ordenamiento jurídico, este asunto en el marco de los debates parlamentarios (Rozenberg, 2010).

Para no entrar en más asuntos que pudieran desviar el presente artículo de lo principal, en los precedentes se extractaron y recogieron todas las menciones aparecidas en los Diarios de Sesiones y también los expedientes relativos al asunto conservados en el Archivo del Congreso de los Diputados. Bien es cierto que en este caso, y como ya se ha observado, no se trata de precedentes legislativos stricto sensu, pero sí que la documentación presentada a modo de precedente cumple el objetivo de presentar el desarrollo histórico y la sensibilidad hacia el asunto manifestada por las Cortes a lo largo de su historia.

Sin embargo, lo sucedido con la tramitación legislativa de la ley en materia de concesión de la nacionalidad española a los sefardíes originarios de España no es, en modo alguno, lo más habitual. Es mucho más corriente que se legisle sobre algo que ya ha sido tratado en el ordenamiento jurídico con anterioridad. Así sucede, por ejemplo, con las modificaciones parciales o totales introducidas en los códigos civil y penal. En este caso los precedentes tienen mayor sistematicidad, pues primero se recopilan y ordenan los expedientes relacionados con las tramitaciones previas, ya sean totales o parciales, de la norma efectuadas con anterioridad y se extractan los debates sostenidos al respecto y recogidos en los Diarios de Sesiones. Este trabajo guarda una cierta similitud con el denominado análisis de las normas que ofrece el Boletín Oficial del Estado, si bien incluye tanto lo que se incorporó finalmente a ellas como aquellas otras enmiendas o propuestas que no llegaron a más.

Analizado, pues, el caso de los precedentes cuando en sentido estricto no existen y aquellos casos en los que sí, procede analizar cuándo y para quién se elaboran los precedentes. Aquí es necesario recordar que los servicios documentales de los parlamentos, y también es así en el caso del Congreso de los Diputados, atienden en primer lugar al trabajo que se lleva a cabo dentro de la cámara. Es, pues, en primer lugar, un servicio interno, cuyo destinatario prioritario son los diputados y personal de los grupos parlamentarios, así como los órganos que rigen su actividad y la Secretaría General.

Partiendo de esta salvedad es más sencillo entender que los precedentes se elaboran para la Mesa, la Presidencia, las Comisiones o el Pleno, los diputados y los grupos parlamentarios, si es que estos tramitan las peticiones de sus señorías, y el personal al servicio de la Secretaría General.

Los precedentes se suelen elaborar, como regla general, a demanda, es decir, previa petición, si bien en ocasiones y debido a la relevancia que tenga la tramitación legislativa que se va a acometer, se pueden elaborar e incluir en el dossier que elabora el Departamento de Documentación. Esto conduce a concluir que la tarea documental de la elaboración del precedente es, casi, artesanal, pues se hace a la medida de las necesidades y se plantea un producto documental ad hoc, también su forma final dependerá de las necesidades. Así, puede incorporarse a uno de los dosieres elaborados por el Departamento de Documentación, que se analizarán por extenso más tarde, presentarse en forma de conclusiones extractando la información de los documentos o, bien, presentar los propios documentos.

Por esta misma razón no son un producto documental preconcebido, sino que, su propia naturaleza aconseja adaptarlos a las necesidades formuladas. Tampoco su estructura puede fijarse de antemano, pues el tipo de norma, su rango, que existan o no normas previas o la naturaleza misma del asunto pueden aconsejar tratar el precedente de un modo u otro, lo que encorsetaría en producto de tener que adaptarse a un modelo preestablecido. Por otra parte, esto no implica que no se trate de un producto documental anárquico o poco riguroso, más bien lo contrario: su peculiaridad hace que cada vez se afronte atendiendo a lo demandado por quien lo solicita. Dicho de otro modo, la flexibilidad es, en este caso, una virtud que 
asegura el cumplimiento de las necesidades de información planteadas por el destinatario.

\subsection{El precedente parlamentario o reglamentario}

No menos importante es el precedente reglamentario o parlamentario, aquel que sirve para conocer cómo se llevó a cabo una determinada cosa o cómo se interpretó el reglamento en ocasiones anteriores. Este caso no afecta directamente a la materia legislativa, pero tampoco resulta por completo ajeno.

Como ya se ha indicado, este tipo de precedentes guardan cierta similitud con el precedente administrativo, por tanto que se busca, para la toma de decisiones, ejemplos previos de actuación en circunstancias similares. En ambos casos también, el precedente administrativo y el reglamentario no ligan la actuación futura en el mismo sentido, pero sí que dan una pauta. Apartarse de la línea de actuación, separarse en definitiva del precedente, no es en modo alguno imposible, si bien, al menos en el caso del precedente administrativo, lleva aparejada la necesidad de justificar en modo debido la actuación.

Este tipo de precedentes son habitualmente requeridos por los Letrados de las Cortes Generales, cuerpo que tiene encomendadas las funciones relativas al asesoramiento jurídico de los órganos superiores de la cámara, y por las direcciones que tienen mayor vinculación con el trabajo parlamentario; a saber: la Dirección de Asistencia Técnico Parlamentaria y la Dirección de Comisiones. Estos precedentes no solo se elaboran a demanda y tras recibir la petición del usuario, sino que permiten de alguna forma anticipar las necesidades y preparar de antemano soluciones documentales.

El Departamento de Archivo, en este sentido, desarrolla una actividad que casi se podría relacionar con la de vigilancia tecnológica, valga la analogía, si bien circunscrita al ámbito parlamentario. La vigilancia tecnológica, tal y como se define en la norma UNE 166000:2006, es el proceso organizado, selectivo y sistemático, llevado a cabo para captar información del exterior y de la propia organización sobre ciencia y tecnología, seleccionarla, analizarla, difundirla y comunicarla para convertirla en conocimiento con el fin de tomar decisiones con menor riesgo y poder anticiparse a los cambios. Asimismo, se entiende este concepto como parte del llamado proceso de Inteligencia Colectiva. A grandes rasgos, el proceso de inteligencia competitiva y el estudio reflexivo y permanente sobre los precedentes en el ámbito de una organización como el Congreso de los Diputados tienen varios puntos en común, pues si bien limitado a su ámbito de actuación, los precedentes se presentan como una herramienta válida para el conocimiento de la propia organización de cara a optimizar el proceso de toma de decisiones y contar con un conocimiento preciso de la propia actuación que permita adelantar los problemas o las situaciones para prever soluciones o modos de actuación.

Atendiendo a esto se preparan, al comienzo de la legislatura, precedentes que puedan servir para la composición de las nuevas comisiones, celebración de las sesiones constitutivas, composición de los órganos superiores del Congreso de los Diputados y situaciones que caben dentro de lo establecido en el Reglamento de la cámara. Para ello se elaboran y actualizan precedentes relativos a todas estas cuestiones que puedan contener referencias precisas sobre el proceder previo y favorecer el proceso de toma de decisiones en ciertos extremos. De otra parte, y en lo que esto afecta a la dimensión externa, gran parte de esta información se ha sistematizado y puesto a disposición de cualquier usuario a través de la página web del Congreso de los Diputados.

El Departamento de Archivo ha contribuido, y lo sigue haciendo de forma permanente, a facilitar información debidamente presentada y contextualizada que sirva para ilustrar el pasado y el presente de la cámara. La información sobre los órganos del Congreso de los Diputados, que abarca desde la Legislatura Constituyente (1977-1979) hasta nuestros días, presenta las funciones, composición, fechas de alta y baja de sus miembros, así como la principal actividad de la Mesa, junta de Portavoces, Diputación Permanente, Comisiones y Subcomisiones y Ponencias. Dentro de Historia y Normas se condensa toda la información relativa a las relaciones entre la Corona y las Cortes, El Congreso entre 1977 y 2017, Presidentes del Congreso de los Diputados (1810-2016) y Constituciones Españolas. También es posible conocer el nombre de todos los diputados, ya sean anteriores a 1977 a través del Fichero Histórico de Diputados o desde entonces hasta los actuales miembros de la cámara a través del apartado "Diputados" del menú principal.

Dicho de otra forma, la relevancia que esta información tiene ha propiciado que se hayan invertido los recursos precisos para ponerla a disposición pública a través de la página web; y el elevado número de visitas a estas secciones da fe de lo acertado de la decisión. Esto también sigue propiciando que periódicamente se actualicen estos contenidos para mantenerlos al día y que puedan seguir siendo una fuente de información fiable y eficaz cuyo uso y reutilización están tan solo limitados por las condiciones que se especifican en el aviso legal de la página web del Congreso de los 
Diputados: que el contenido de la información no sea alterado, que no se desnaturalice el sentido de la información, que se cite la fuente, que se mencione la fecha de la última actualización y que se siga un principio de acceso público y de no exclusividad. Estas son algunas de las materializaciones de la actividad de elaboración de precedentes parlamentarios llevada a cabo por el departamento que trasciende la labor que se desarrolla al servicio del propio Congreso de los Diputados y que se pone al alcance de cualquiera que desee acercarse a la actividad de la cámara a lo largo de su Historia y también en el presente.

\section{El Departamento de Documentación y la serie legislativa}

El objeto de cualquier centro de documentación es ofrecer servicios de información acordes a las necesidades reales de la institución a la que pertenecen y a sus usuarios directos. Así, el Centro de Documentación del Congreso de los Diputados apoya al desempeño de la actividad parlamentaria, entre otros servicios, mediante la edición de publicaciones electrónicas y, ofrece a la función legislativa, un instrumento documental adecuado para la elaboración de las leyes: los dosieres legislativos.

El Departamento de documentación del Congreso de los Diputados fue creado en 1984 al aprobar la Mesa de la Cámara las Normas sobre los servicios documentales del Congreso de los Diputados. En este momento se estructura en tres servicios: Servicio de Proceso Técnico, Servicio de Tratamiento de los Recursos Documentales y Servicio de Difusión de la Información.

El Servicio de Difusión se subdivide en las siguientes áreas temáticas: documentación española y bibliográfica, documentación europea y documentación extranjera y de organismos internacionales. A través de estas áreas se pretende canalizar y difundir información sobre legislación, doctrina, jurisprudencia y cualquier otro tipo de recurso documental de una manera transversal en cualquier materia que requiera el usuario.

Sus servicios son fundamentalmente dos: la atención a las peticiones de información y documentación sobre legislación, jurisprudencia, bibliografías, artículos de revista, prensa, derecho comparado y otro tipo de documentos que puedan ser de interés en las áreas antes citadas en los campos de interés tanto para los diputados, grupos parlamentarios o personal de la cámara; y la realización y edición de boletines y dosieres. Edita tres series: la serie legislativa - cuyo contenido se ajusta a cada proyecto de ley recibido en la Cámara, objeto de esta presentación y que explicaremos a continuación, la serie general en función de las necesidades de la cámara y de las comisiones-, los boletines de novedades - por cada área citada se ofrece de manera periódica una selección de documentos recientes-y el Boletín de Información Parlamentaria, con la actividad semanal de la Cámara.

Como ya hemos dicho la serie legislativa está compuesta por los dosieres producidos por cada Proyecto de Ley recibido. Entre las tareas antes citadas tiene carácter prioritario puesto que debe finalizarse y publicarse antes del final del periodo de enmiendas. La elaboración de este tipo de dosieres se remonta al año 1983 con el dosier número 1 sobre las Comunidades Autónomas.

La adaptación y la sistematización de un modelo práctico de gestión de la documentación son las claves que han demostrado que el dosier legislativo es un producto viable y fiable que es demando como herramienta de trabajo. El desarrollo, muy influenciado por la tecnología, se ha orientado en dos direcciones: la informativa (documentos comparados, actualización y nuevas fuentes) y la editorial con la producción de distintos formatos para su consulta.

Podríamos decir que nuestro trabajo es el entresuelo del procedimiento parlamentario, de la publicación del proyecto a su tramitación se edita un producto que analiza y entresaca las características principales dadas en el texto presentado por el Gobierno antes de su tramitación. Hacemos nuestros los principios de buena regulación el artículo 129.1 de la Ley 39/2015 para una buena asistencia documental parlamentaria ofreciendo un producto necesario que cita y enlaza a texto completo la normativa citada en el texto, de forma integrada y publicado través del Portal de Transparencia de la cámara.

En la actualidad, por una cuestión de eficacia y eficiencia, se realizan exclusivamente dosieres de proyectos de ley; ya que, como se ha mencionado anteriormente, el Servicio de Difusión realiza otras funciones de apoyo al desempeño de la actividad parlamentaria y los recursos son limitados lo que evidentemente restringe la capacidad de respuesta entre la multitud de proposiciones de ley que se presentan; por ejemplo se pueden tomar los datos de la $X$ legislatura: 163 proyectos de ley ante 217 proposiciones de ley de Grupos Parlamentarios del Congreso; realizar el proceso que hoy presentamos por cada proposición de ley es, en estos momentos inabarcable. Además, a esta cuestión habría que añadir la premura de los plazos de tramitación parlamentaria, especialmente el de urgencia, que obliga a tener que excluir, en determinadas ocasiones, algunos de los proyectos de ley presentados. Y, sin embargo, volviendo 
a las cifras se presentaron un total de 109 dosieres de la serie legislativa en la $\mathrm{X}$ legislatura.

\subsection{El dosier legislativo: metodología y fases}

La metodología de trabajo no deja de constatar la importancia de los procesos documentales normalizados y flujos de trabajo sistemáticos en nuestro campo que pretenden hacer accesible la información. Para la elaboración de los dosieres legislativos estos procesos normalizados suponen un proceso de lectura detenida, con el consabido proceso de análisis documental como descripción de contenido. Pero, en este caso, nos encontramos, también con las peculiaridades de la técnica normativa respecto a la estructura, la redacción y el lenguaje. Por lo que, en muchos casos, se impone la necesidad de realizar relecturas para poder llegar a un proceso de abstracción que permita redactar una presentación objetiva y sintetizar y ofrecer los puntos de referencia correctos. Por otra parte, la secuencia de tareas y objetivos que se establecen sigue una serie de estándares marcados por los plazos parlamentarios y por las fases documentales propias de elaboración, la revisión y la edición. Como se desarrolla a continuación, cada una de las fases determina una serie de acciones y procesos tanto documentales como administrativos que conforman un producto informativo-analítico a favor de la actividad parlamentaria, que hoy alimenta de contenido al Portal de Transparencia.

El punto de partida de este proceso documental es la recepción del texto del Proyecto del Ley enviado por la Secretaría de Asistencia Técnico Parlamentaria, en el que se incluye, además, la memoria de impacto normativo y otros documentos como memorias, informes y dictámenes preceptivos o no- que se hayan adjuntado en la remisión el mismo. El procedimiento comienza con tareas administrativas propias de la gestión documental, en la que se comprueba el tipo de tramitación y los plazos, que determina la realización del dosier. En caso afirmativo, se continúa con el procedimiento habitual; en caso negativo - como veremos más adelante- si se incluye una nota en el Portal de Transparencia con las referencias y documentos complementarios que acompañan al Proyecto de ley.

Como se ha mencionado anteriormente, el procedimiento habitual para el análisis documental implica la lectura completa del documento. Esta peculiaridad responde al valor informativo de todo el texto normativo y de las referencias de los documentos que lo acompañan; por eso, se divide el trabajo entre las tareas de análisis, selección y búsqueda. Aun así, debido a su naturaleza compleja, no se puede analizar toda su casuística, pero sí describir los procedimientos y fuentes generales que utiliza, siguiendo la estructura general establecida para la elaboración del dosier.

\subsection{Partes del dosier}

El dosier, normalmente incluye los siguientes apartados: documentación que acompaña al proyecto de ley, legislación española, documentación complementaria, de la Unión Europea, de organizaciones internacionales, derecho comparado, y estudios. A continuación, analizaremos cada uno de estos apartados, haciendo referencia a su contenido y las fuentes de información más utilizadas.

Comenzamos hablando del texto del Proyecto de Ley y la documentación que lo acompaña. Inicia todos los dosieres de esta serie. Contiene el enunciado de la iniciativa, el enlace a su publicación en el Boletín Oficial y el propio a la tramitación parlamentaria, de tal manera que el usuario, en cualquier momento, pueda acceder a la ficha del iter parlamentario. Además, se insertan los documentos que acompañan preceptivos o no, a dicho texto. De manera habitual, por su obligatoriedad, es la Memoria del Análisis de Impacto Normativo y los informes y dictámenes preceptivos establecidos por el ordenamiento jurídico como son aquellos que provienen del Consejo de Estado. Si bien no son objeto de este artículo debemos remitir para su estudio a los diversos comentarios sobre el artículo 88 de la Constitución Española, al Real Decreto 931/2017, de 27 de octubre, por el que se regula la Memoria del Análisis de Impacto Normativo y a todas aquellas leyes de órganos constitucionales o estatales que determinen entre sus funciones la consultiva con este fin.

A continuación le sigue la presentación del dosier que indica el objeto de estudio, señala el marco normativo y la metodología a seguir en el análisis del texto legislativo. Esta breve referencia no es una anécdota en la nota que hoy presentamos puesto que el texto introductorio pretende ser un resumen del proyecto de ley y una guía práctica para la consulta del producto documental que se presenta.

Después de la introducción se desarrollan los apartados resultados del análisis del texto: su primer apartado, es el dedicado a Documentación española, que se divide en las áreas de legislación citada, jurisprudencia citada, documentos comparativos y documentación complementaria.

Por las técnicas de redacción normativa, la gran mayoría de las referencias a la legislación y a la jurisprudencia, se concentran principalmente en la Exposición de motivos y en los antecedentes pero se revisa todo el articulado para entresacar 
dichas referencias como citadas. Evidentemente, las fuentes deben ser públicas, oficiales y/o que cumplan con las obligaciones de publicidad activa; por tanto nos referimos al BOE, los boletines oficiales de cada una de las CCAA, la base de datos del CENDOJ y los apartados de publicaciones y normativa de Ministerios, gobiernos autonómicos, etc. El orden habitual comienza con la cita de los artículos de la Constitución Española $y$, después las normas por orden ascendente de fecha de publicación, prefiriéndose los textos consolidados y el formato pdf. Se dan casos de reorganización de este esquema, por ejemplo, cuando se realizan subsecciones para los convenios y tratados internacionales o la legislación autonómica en el apartado de legislación citada.

Los documentos comparativos reflejan la indicación de adiciones, modificaciones y derogaciones de otras normas legales en relación con el texto presentado, se incluya en el título de la ley - si es su objeto- o en las disposiciones finales. Se realiza una comparación entre el texto vigente y las propuestas que se plantean: derogación, adición y modificación. Este tipo de documentos resaltan por añadir valor analítico al dosier e informa muchas veces del objeto de la ley. Su elaboración artesanal requiere establecer adecuadamente el contexto de la norma vigente. Por tanto, su importancia radica en señalar el antecedente de la norma que se desarrolla con el nuevo proyecto.

La documentación complementaria es un subapartado que podremos incluir tanto en las secciones de Unión Europea, Documentación Extranjera, Organismos Internacionales o añadirlo a los márgenes. La tipología documental de los documentos es, en su gran mayoría, literatura gris - quizá un título poco acertado hoy para el desarrollo en sus canales de difusión-. Dentro de este grupo encontramos una gran diversidad de tipos documentales (entre otros, pre-prints, actas, comunicaciones, trabajos, opiniones, informes y documentos de trabajo) de instituciones diversas, públicas o privadas o referenciadas en bases de datos generalistas o de investigación. Su inserción el dosier debe estar justificada y ceñirse exclusivamente al tema de interés para incrementar la información final al usuario.

La siguiente gran referencia que encontramos en el dosier se refiere a la Unión Europea. El ordenamiento jurídico comunitario y su aplicación como fuente del derecho en el corpus jurídico interno tiene cada vez más relevancia en el diseño de estos productos documentales. Sus posibles apartados son legislación citada, jurisprudencia citada y documentación complementaria. Su ordenación es cronológica, sus textos legislativos consolidados y las fuentes son las conocidas Eur-Lex, el registro de documentos de la
Comisión, Curia o el Parlamento Europeo, sin despreciar en ningún caso aquellos producidos por agencias o instituciones oficiales comunitarias.

A continuación se desarrolla el apartado de Documentación extranjera, probablemente el apartado más complejo. Si bien algún documento o informe que acompaña al proyecto de ley puede citar algún caso en derecho comparado la inclusión de este apartado supone criterios de selección, búsqueda y presentación de la información de forma adecuada y objetiva. Debemos destacar que la traducción jurídica de los conceptos implica un conocimiento del contexto y de la organización jurídico-administrativa de cada país, por tanto, requiere, en la mayoría de las ocasiones un estudio previo y la consulta de diccionarios, tesauros y otros documentos que ofrezcan fiabilidad. Normalmente, se escogen países del entorno geográfico y/o jurídico $\mathrm{y}$, dependiendo del tema principal, se amplía el abanico; por ejemplo en el caso del dosier dedicado al Proyecto de Ley de Patentes se incluyó legislación de Estados Unidos, o en el dedicado a Proyecto de Ley de salvaguardia del Patrimonio Cultural Inmaterial se citaron normas de Argentina, Brasil, Guatemala y Japón, entre otros. En algunos casos, los menos, este apartado no se incluye por ser objeto de aplicación de una directiva comunitaria.

Respecto a la selección de las fuentes se utilizan siempre recursos oficiales de legislación y publicación de diarios oficiales, sedes parlamentarias $y$ organismos oficiales.

El ámbito internacional se completa con el apartado de Organismos Internacionales. En primer lugar, debemos observar que se restringe el concepto de este tipo de organismos a aquellos que se forman por una asociación de estados, que gestionan intereses colectivos con autonomía jurídica y sujetos al derecho internacional de aquellas otras organizaciones voluntarias internacionales. Algunos ejemplos son el Consejo de Europa -y por extensión el Tribunal Europeo de Derechos Humanos-, el Banco Mundial, el FMI, la ONU, la OMC o la Organización Mundial de la Propiedad Intelectual, entre otros, que producen una ingente cantidad de documentación que cubre un amplio abanico geográfico y temático, propios de una sociedad globalizada. Si en el apartado de documentación española se recogen los tratados y acuerdos internacionales firmados por España, éste apartado ofrece documentos como acuerdos, resoluciones, informes, guías, acuerdos publicados por este tipo de órganos que expresan el interés del mismo en ciertas áreas. Las fuentes, como no podría ser de otra manera, son las propias bases de datos de estos organismos. 
Como parte de los recursos que se incluyen en los dosieres legislativos, se incluye el apartado de Estudios, también denominado de doctrina. Es una selección de los artículos más recientes y acordes con el tema principal del proyecto de ley. Los fondos citados proceden del catálogo propio, de los recursos electrónicos a los que se está suscrito y, en algunos casos se han incluido referencias a revistas de acceso abierto.

Finalmente, la selección de libros, es una bibliografía elaborada por los compañeros de la biblioteca para cada dosier con enlaces directos a su ficha catalográfica.

El único apartado que permite cierta creatividad es el denominado Para saber más, que se encuentra en los márgenes. Aquí se incluyen enlaces a organismos públicos o privados y documentos de think tanks, citas y definiciones que intentan ofrecer un cierto dinamismo al dosier.

\subsection{Edición y presentación}

Una vez concluida la labor preparatoria y la redacción de la presentación se realizan las correcciones pertinentes tras diversos procedimientos de revisión -que llegan a la Dirección de Archivo, Biblioteca y Archivo-. La edición en formatos papel, CD y electrónica, tanto en edición intranet como transparencia, implica revisión de concordancias, tareas administrativas, de reproducción y difusión donde se implica todo el personal del departamento.

Entre los formatos citados destacamos su publicación en el Portal de Transparencia del Congreso de los Diputados bajo el epígrafe Documentación complementaria de las iniciativas legislativas al final del apartado dedicado Información institucional y organizativas. El primer dosier publicado fue el $n^{\circ} 68$ de la $X$ Legislatura dedicado a la Jurisdicción voluntaria con fecha de diciembre de 2014. En la X Legislatura se publicaron 42 dosieres y, en la actual, 15.

En todos los casos se realiza lo que podríamos denominar dosier sencillo con la nota introductoria que comprende: el título del proyecto de ley, su texto y documentación complementaria, el texto indicativo sobre su tramitación y los enlaces correspondientes. Si el dosier se realiza en su formato completo se sustituye, donde el único cambio es la supresión de los enlaces a los documentos que provienen de recursos electrónicos de pago. Con esto, el servicio de difusión supera su ámbito previsible para llegar a los usuarios externos - especializados o no-y proporciona contenidos fiables para el estudio de la cada una de las iniciativas, coadyuvando a dar un valor añadido al principio de publicidad parlamentaria

\section{Conclusiones y reflexiones finales}

En las líneas precedentes se ha tratado de describir y sistematizar el análisis de dos productos documentales que ofrecen a la labor legislativa apoyo informativo, ya sea de actualidad o con carácter retrospectivo, con la finalidad de contribuir al eficaz desempeño de dicha actividad.

El precedente elaborado por el Departamento de Archivo es un producto documental de uso habitual y recurrente en el ámbito de la actuación parlamentaria y, en lo que atañe a la producción normativa, un útil auxiliar capaz de proporcionar a su destinatario una visión panorámica y retrospectiva sobre la evolución de un asunto determinado en la legislación previa y también capaz de presentar la sensibilidad del poder legislativo hacia aquel en su tratamiento y debate.

Por su parte, respecto a los dosieres legislativos, preparados por el Departamento de Documentación, se ha presentado el modelo preestablecido de trabajo documental, su ordenación y sus fuentes. Su objeto es presentar el contenido del Proyecto de Ley, identificar las normas que impulsan, sustentan, modifican o se insertan en el articulado del texto normativo tanto españolas como europeas; presentar el contexto normativo con las referencias al derecho comparado y de organismos internacionales y seleccionar estudios y bibliografía que amplíen el conocimiento sobre el tema del dosier, ofreciendo evidentemente, la referencia completa a su fuente. La elaboración de ambos y su difusión a través de diferentes canales pretende ofrecer una herramienta de estudio previo sobre un proyecto normativo que posibilita el acceso directo a los documentos seleccionados en cada uno de ellos. Con esta aproximación a una sistematización de su forma de elaboración, metodología, objetivos y destinatarios se pretende situar el trabajo del ArchiveroBibliotecario parlamentario en el marco general que constituye el procedimiento legislativo.

Asimismo, este repaso por el trabajo desarrollado por los Departamentos de Archivo y Documentación del Congreso de los Diputados en la elaboración de productos documentales de apoyo al desarrollo de la función legislativa puede servir de punto de partida para el análisis de su efectividad y también del modo de ponerlos al alcance de otros usuarios. Los usuarios primeros de estos productos son los diputados, asistentes de los grupos parlamentarios y los Letrados de la cámara. Estos últimos tienen, entre sus funciones, la de asesorar en el ámbito jurídico (antecedentes, doctrina, legislación y derecho comparado) y, muy especialmente, en todas las cuestiones que tienen que ver con el reglamento y su aplicación. En este sentido la labor de los Archiveros-Bibliotecarios se 
presenta como un instrumento útil para el desarrollo de la función legislativa por quienes la tienen encomendada y también para el cuerpo que asesora en esta labor a los diputados.

Como antes se apuntaba, estas tareas guardan ciertos puntos de contacto con los que en el ámbito profesional se ha dado en llamar vigilancia tecnológica, llegando a convertirse en estándar internacional. En este sentido, sería preceptivo hacer un esfuerzo para adaptarse a lo previsto en estas normas, dando el salto hacia nuestra conversión en vigilantes tecnológicos del ámbito parlamentario, adelantándonos a las necesidades de los usuarios y siendo capaces de prever en ciertos aspectos el futuro próximo.

En último término, la materia presentada sugiere una inquietud acerca del papel del archivero-bibliotecario parlamentario en el ámbito de la difusión; en tanto que si se replanteara la posibilidad de llegar a otros usuarios potenciales debe ser bajo el prisma de la publicación en temas de actualidad. Sirva en este sentido el reciente artículo de Inmaculada Moreno a propósito de la gestación subrogada en la legislación internacional. Aceptar como cierta esta posibilidad obligaría a contar con más recursos que hicieran posible extender nuestro trabajo más allá de lo aquí expuesto para poder adelantar las necesidades, acortar los tiempos de edición o puesta a disposición de los productos documentales al público y estar en condiciones de elaborar estudios sobre la práctica profesional, como el que hoy se presenta, con el objetivo de dar a conocer el trabajo en un ámbito muy desconocido que, sin embargo, cuenta con una larga tradición.

En última instancia, es preciso aludir a la necesidad, en un mundo cada vez más interconectado y en el que las barreras de la información son más imprecisas, de establecer sinergias y fortalecer la cooperación entre todos los sectores implicados en la difusión de la información. Así, los centros de documentación, archivos y bibliotecas deben, en la medida de lo posible, estrechar sus lazos y que la colaboración entre ellos sea parte de su cultura organizacional.

Querríamos cerrar ya esta comunicación tomando prestada una vez más la cita de Bartolomé José Gallardo que inauguraba estas líneas para afirmar, sin miedo a equivocarnos, que hoy, como en el siglo XIX, para elaborar buenas leyes son menester, más que nunca, la información adecuada y objetiva que brinda el centro de documentación, los libros y las bibliotecas y la memoria fiel que se guarda en los archivos.

\section{Referencias}

Bartolomé, José Gallardo (1838). Adiciones y refundición de algunos títulos y artículos del Proyecto de Reglamento para el gobierno interior del Congreso, propuestas y motivadas por el diputado B. J. Gallardo, Bibliotecario de Cortes. Madrid: Imprenta de D. M. Calero, 1838.

Estatuto del Personal de las Cortes Generales. Aprobado por las Mesas del Congreso de los Diputados y del Senado en su reunión conjunta de 26 de junio de 1989. // BOE. 117 (26 de julio de 1989).

Gallego, Josefa (2006). Apuntes biográficos y bibliográficos sobre la figura de D. Bartolomé José Gallardo. // Estudios Humanísticos. Historia. 5 (2006) 227-237.

García-Escudero Márquez, Piedad (2005). Nociones de técnica legislativa para uso parlamentario. // Asamblea: Revista Parlamentaria de la Asamblea de Madrid.13, 121-164.

García Sánchez, M. a Isabel (2002). El Departamento de Documentación del Congreso de los Diputados y la documentación parlamentaria. // Revista general de información y documentación. 12:1, 163-174.

Grau Guadix, Rosa María (2010) Documentación y parlamento: evolución organizativa y perspectivas de futuro. // Las direcciones de estudios parlamentarias: [Seminario celebrado en Vitoria-Gasteiz los días 7 y 8 de octubre de 2008].Vitoria-Gasteiz: Eusko Legebiltzarra = Parlamento Vasco, 2010. ISBN 978-84-87122-97-2. 115-149.

Maciá, Mateo; Valle de Juan, Ma Ángeles (2002). Los archivos del poder legislativo. // Revista general de información y documentación. 12: 1 (2002) 13-32.

Martín González, Alicia; García de la Oliva, África (1986). Organización de los servicios documentales en el Congreso de los Diputados. // Boletín de la ANABAD. 36:1-2, 345-352.

Moreno de la Fuente, Inmaculada (2017). La gestación por sustitución: Derecho internacional y Derecho comparado. // Revista general de derecho constitucional. ISSN 18866212. 25, 2017.

Normas de la Mesa del Congreso de los Diputados, de 20 de enero de 2015, para la aplicación de las disposiciones de la Ley 19/2013, de 9 de diciembre, de transparencia, acceso a la información pública y buen gobierno a la Cámara, en relación con su actividad sujeta a derecho administrativo. // BOCG. 595, Serie D ( 23 de enero de 2015).

Proyecto de Ley de Patentes. // Dosieres de la Serie Legislativa. X Legislatura, 71 (febrero 2015). Congreso de los Diputados. Portal de Transparencia. http://www.congreso.es/docu/docum/ddocum/dosieres/sleg/legislatura 10/spl_71/dosier_sl_71_patentes_electronico.pdf

Proyecto de Ley de salvaguardia del Patrimonio Cultural Inmaterial. // Dosieres de la Serie Legislativa. X Legislatura, 71 (febrero 2015). Congreso de los Diputados. Portal de Transparencia. http://www.congreso.es/docu/docum/ddo cum/dosieres/sleg/legislatura_10/spl_70/dosier_sl_70_ patrimonio_electronico.pdf

Rozenberg, Danielle (2010). La España contemporánea y la cuestión judía. Madrid: Marcial Pons, 2010.

Enviado: 2018-03-28. Segunda versión: 2018-06-15. Aceptado: 2018-09-05. 